\title{
Ectopic gall bladder - An interesting case report
}

\section{Sharmin Reza, Faria Nasreen, Sharmin Quddus, Tapati Mandal, Ferdous Ara Hussain}

National Institute of Nuclear Medicine and Allied Sciences, BAEC, BSM Medical University Campus, Dhaka.

Address for correspondence: Dr. Sharmin Reza, Senior Medical Officer, National Institute of Nuclear Medicine and Allied Sciences, BSMMU campus, Dhaka. E-mail: rezasharmin19@gmail.com

\begin{abstract}
Ectopic gall bladder is a rare entity. It can often be misdiagnosed causing diagnostic dilemma leading to various complications. Ultrasonography is the most common investigation for evaluating gall bladder pathologies. However, the confirmation of ectopic gallbladder is not easily possible by this method. On the other hand, hepatobiliary scan (HIDA scan) plays an important role in evaluating the presence and position of ectopic gallbladder. Here we present a case of sonographically suspected ectopic gallbladder confirmed by hepatobiliary scan highlighting the importance of HIDA scan in ectopic gallbladder.
\end{abstract}

Key words: Ectopic gall bladder, Ultrasonography, HIDA scan.

\section{INTRODUCTION}

Ectopic gall bladder is a rare entity. Its incidence being $0.1 \%-0.7 \%$ (1). The normal gall bladder lies in the gall bladder fossa on the inferior surface of the liver in between the right and left hepatic lobes maintaining a constant relationship to portahepatis (2). On Ultrasonographic imaging gall bladder can be identified by the relationship of the gall bladder neck anterior to the right branch of the portal vein. Ectopic gall bladder can be located in various positions such as, intrahepatic, within the lesser omentum, retroduodenal, within falciform ligament, abdominal wall muscles and in thoracic cavity $(3,4)$. An ectopic gall bladder is a dangerous entity as it can lead to misdiagnosis.

We present a case of sonographically suspected ectopic gall bladder, which was confirmed by HIDA scan.

\section{CASE REPORT}

A 16 years old male was referred to the National Institute of Nuclear Medicine and Allied Sciences (NINMAS) for ultrasonography of whole abdomen. $\mathrm{He}$ complained of generalized weakness and polyuria. Ultrasonography showed that gallbladder was not in its usual position rather an elongated cystic area was noted in right lateral aspect of right lobe of liver (Figure 1), which seemed to be separated from liver and right kidney.

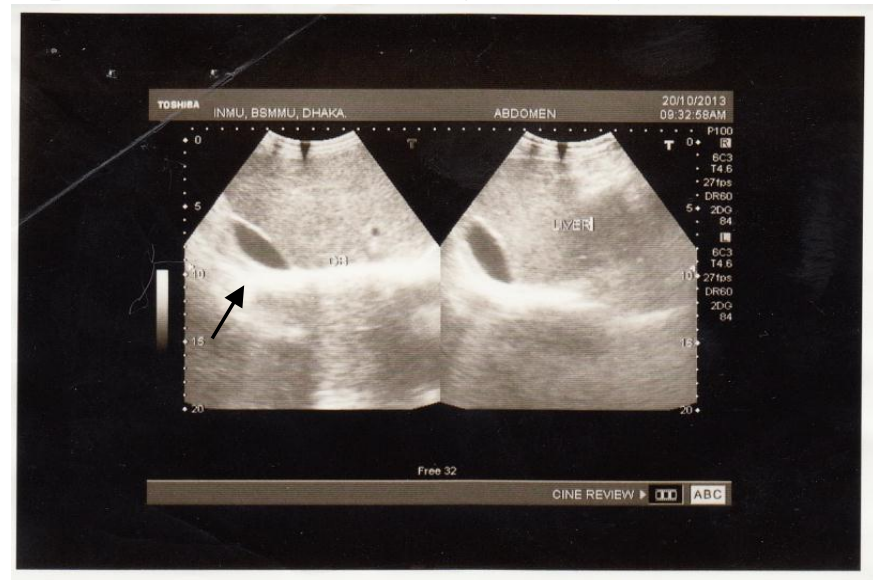

Figure 1: Ultrasonography of liver and gall bladder showing ectopic position of gall bladder

The provisional diagnosis was ectopic gall bladder with a differential diagnosis of right-sided pleural effusion. For further evaluation the patient was given a fatty meal. Ultrasonography was repeated two hours after intake of fatty meal, which showed decrease in the size of the cystic area indicating the contraction of ectopic gallbladder (Figure 2).

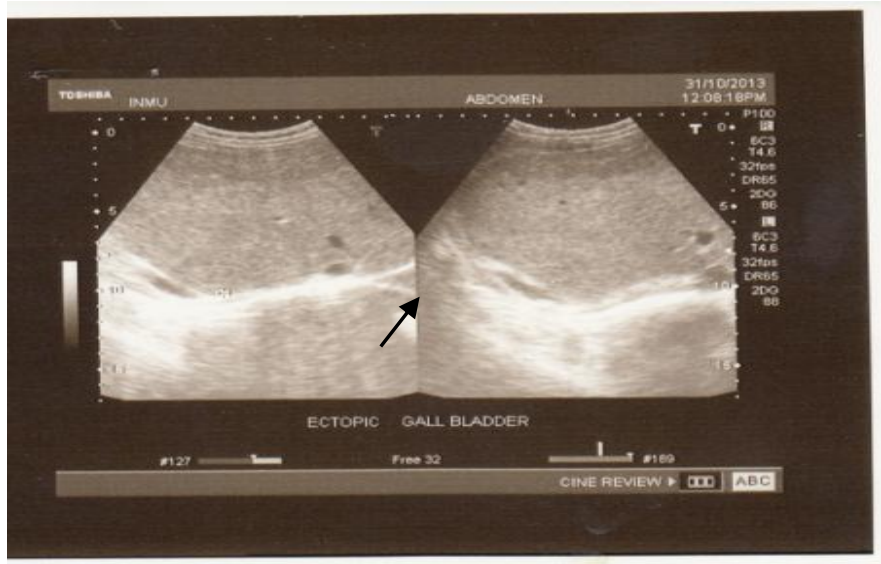

Figure 2: Ultrasonography image showing postfatty meal contraction of ectopic gall bladder. 
For further confirmation a hepatobiliary scan was performed using Tc-99m Bromo 2,4,6 trimethylacetanilido iminodiacetic acid (BrIDA) as the radiopharmaceutical to diagnose ectopic gallbladder. Patient fasted for 3-4 hours before the study. $10 \mathrm{mCi}$ of $\mathrm{Tc}-99 \mathrm{~m}$ labeled BrIDA was injected intravenously to the patient. One static view was taken immediately after injection followed by 5 minutes images taken at interval upto 60 minutes. At 60 minutes right lateral and left anterior oblique images were taken. In this case, hepatobiliary scan showed (Figure 3) uniform radiotracer concentration in the liver.

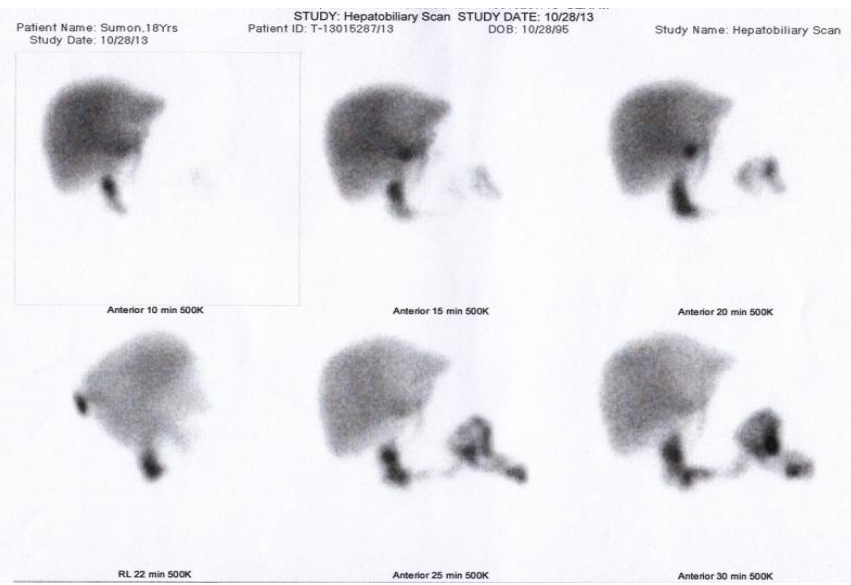

Figure 3: HIDA scan showing ectopic position of the gallbladder

Common bile duct was visualized at 5 minutes image. Gallbladder was not seen in its original position rather a small, elongated area of tracer accumulation was seen in the posterolateral aspect of right lobe of liver in the right lateral view at 22minute image (Figure 4), which represented the ectopic gall bladder.

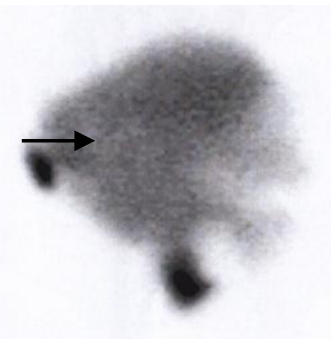

Figure 4: HIDA scan showing ectopic position of the gallbladder

\section{DISCUSSION}

The gallbladder is generally found in the right upper quadrant of abdomen situated in segments IVB and $\mathrm{V}$ of the liver (5). Anatomically, the gallbladder is divided into three sections: the fundus, body and neck. The gallbladder measures approximately 8 centimetres in length and 4 centimeters in diameter when fully distended (6). Gallbladder has three types of congenital anomalies- anomalies in shape, anomalies in location and congenital absence. An ectopic gallbladder is one, where gallbladder is not located in its usual anatomical position rather it is seen in an unusual site on routine imaging. Ectopic gallbladder may cause clinical symptoms or may be asymptomatic. If ectopic one is situated away from peritoneum, typical signs and symptoms of acute cholecystitis may be absent. The ectopic gallbladder may be floating suspended from a long mesentery, which is susceptible to torsion and gangrene (7). Peristaltic movements of the stomach, duodenum, and the transverse colon can cause torsion of the ectopic gallbladder. Floating gallbladder may also herniate through the foramen of Winslow into the lesser sac. Other diagnostic modalities to locate the position of gallbladder are computerized tomography (CT), ERCP and radionuclide imaging (8). These modalities can diagnose gallbladder position and location. Radionuclide imaging of hepatobiliary system commonly known as HIDA scan is used routinely for diagnosing various acute and chronic hepatobiliary diseases including acute or chronic cholecystitis , ectopic gallbladder, biliary atresia, biliary obstruction, bile leakage (9). In the present case, gall bladder was not identified in its original position during ultrasonography, rather an elongated cystic structure was seen in right lateral position of right lobe of liver mimicking pleural effusion. Fatty food caused contraction of this cystic area. Tc-99m labeled HIDA scintigraphy was performed to confirm the presence and the position of the gall bladder. HIDA scan showed small 
radiotracer accumulation in the postero-lateral aspect of right lobe of liver and confirmed the ectopic gallbladder.

\section{CONCLUSION}

Ectopic gallbladder can raise diagnostic dilemma. Hepatobiliary scan plays an important role in diagnosing various gallbladder anomalies including agenesis and ectopic position at the expense of minimum radiation exposure.

\section{REFERENCE}

1. Rather AT, Khan HS, Singh M, Choh AN. A Rare Case of Suprahepatic Gall bladder with Phocomelia and Pancytopenia: Detected by Tc99m Mebrofenin Scintigraphy. World Journal of Nuclear Medicine 2013; 12:41-44.

2. Meloughlin JM, Fanti EJ, Kura LM. Ectopic gallbladder: Sonographic and scintigraphic. The Journal of Clinical Ultrasound 1987; 15(4): 258-61.

3. Popli MB, Popli V, Solanki Y. Ectopic Gall Bladder: A Rare Case. The Saudi Journal of Gastroenterology2010;16(1):50.

4. Audi P, Noronha PF, Rodrigues J. Intrahepatic gallbladder- A case report and review of literature. The internet journal of surgery 2009 ; 24(1): 167-72.

5. Dever RC. Suprahepatic gallbladder with torsion and gangrene. J Fla Med Assoc 1968; 55:531.

6. Bockman DE, Freeny PC: Anatomy anomalies of the biliary tree. Laparos Surg 1992; 1: 92.

7. Agarwal V, Pande S, Garg SK, Jangid DR. Anomalously placed suprahepatic gall bladder: A case detected on F-18 FDG PET/CT. Indian J Nucl Med 2011; 26:120-2.

8. Maeda N, Horie Y, Shiota G, Suou T, AndachI $\mathrm{H}$, Kawasaki H. Hypoplasia of the left hepatic lobe associated with floating gallbladder: A case report. Hepatogastroenterology 1998; 45:1100-3.

9. Mcnamee EP: Intrahepatic gallbladder. Am J Roentgenol; Radium Therapeutic Nucl Med 1974; 121: 396-400. 